\title{
IoT Based Silence Monitoring System
}

\author{
E. Esther Devakirubai ${ }^{1}$ and G. Indirani ${ }^{2}$ \\ ${ }^{1}$ PG Student, Department of Electronics and Instrumentation Engineering \\ ${ }^{2}$ Assistant Professor, Department of Computer Science Engineering \\ ${ }^{1 \& 2}$ Annamalai University, Chidambaram, Tamil Nadu, India \\ E-Mail: estherdvkrb@gmail.com, induk0992@gmail.com
}

\begin{abstract}
Internet of Things (IoT) finds application in many fields these days. This paper focuses on the use of IoT based monitoring of Silence which can be used in places where Silence maintenance is important like in libraries and in classrooms of schools. Further this paper proposes a system based on Arduino which involves programming Arduino Uno to achieve the purpose of Silence monitoring.
\end{abstract}

Keywords: Silence Monitoring, Arduino, IoT

\section{INTRODUCTION}

The proposed work uses an Arduino Uno board which is programmed in such a way that it senses the sounds and when the sensed input is higher than the threshold value an alert system is activated. This system can be used in libraries. Since libraries cannot allow noises, a flash light system can be used as alert system. This can also be used in Classrooms of schools to monitor silence of classrooms at the absence of the teacher. These days Schools maintain a record called Silence record in which at the end of each period, the teacher used to sign and make a remark on how the silence is maintained in his/her class. The proposed system can replace the existing system. When the intensity of the sounds produced is higher than the threshold intensity, then some Siren or bell which has a sound intensity capable of drawing the attention of the children but without being a disturbance to the neighbours is activated. Hospitals can also make use of the same system but the warning systems should be properly designed such that it does not affect the patients.

\section{MODULES USED}

\section{A. LM393 Sound Detection Sensor}

Fig. 1 shows the LM393 Sound detection sensor.

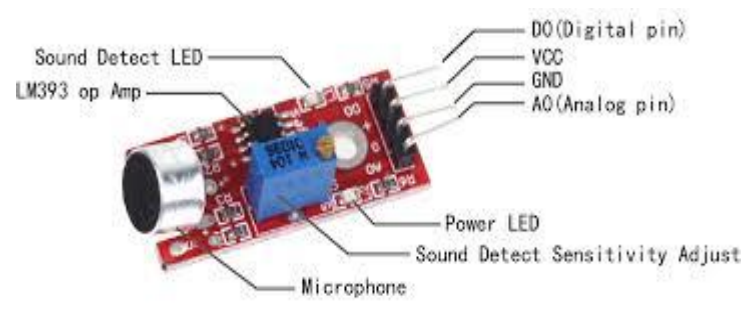

Fig. 1 LM393 Sound Detection Sensor

It is very easy to detect sounds using the sound sensor module and is generally used for detecting sound intensity.
This module can be used for switching, monitoring and security applications. For the convenience of usage, its accuracy can be easily adjusted. This Sensor integrates a microphone, a sound Detect Sensitivity adjust and LM393 Operational Amplifier along with some LEDs for the indication of Power and detection of sound. This module has four Pins which are for Ground, 5V DC Supply, analog output pin and digital output pin. The LM393 Op-amp amplifies the input received by the microphone. The output of this module is sent to the Arduino Uno which acts as the microcontroller that is capable of doing the further processing.

\section{Features of LM 393 Sound Detection Sensor}

a. LM 393 sound detection sensor has four pins.

b. Both digital and analog outputs can be obtained.

c. Contains an electret condenser microphone.

\section{Electrical Characteristics}

Working Voltage: $3.3-5 \mathrm{~V}$

\section{B. ESP $8266 \mathrm{Wi}$-Fi Module}

The ESP8266 is one of the cost-effective Wi-Fi microchips with full TCP/IP stack and microcontroller capability. It works with a voltage supply of $3.3 \mathrm{~V}$. Fig. 2 shows the ESP $8266 \mathrm{WiFi}$ module.

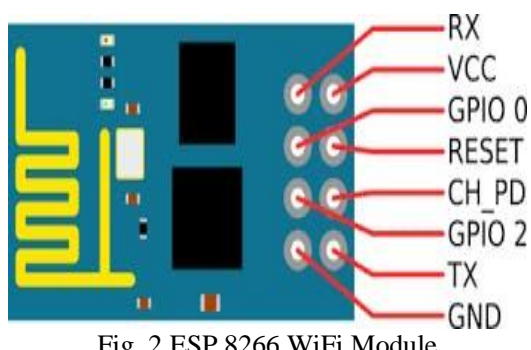

The ESP $8266 \mathrm{WiFi}$ module is a self-contained SOC with integrated TCP/IP protocol stack that can give any microcontroller access to the WiFi network. The ESP8266 is capable of either hosting an application or offloading all Wi-Fi networking functions from another application processor. Each ESP8266 module comes pre-programmed with an AT command set firmware and can be easily hooked up to the Arduino device. 


\section{A. Features of ESP 8266 Wi-Fi Module}

1. Integrated low power 32-bit MCU

2. Integrated TCP/IP protocol stack

3. Wake up and transmit packets in $<2 \mathrm{~ms}$.

\section{B. Electrical Characteristics}

1. Working Voltage: $3.3 \mathrm{~V}$

2. Maximum IO Driving Power IMAX: $12 \mathrm{~mA}$

3. Maximum Voltage level VMAX: $3.6 \mathrm{~V}$

4. Current consumption: $100 \mathrm{~mA}$

This module is used to send data obtained by the sensor to the cloud.

\section{PROCESS FLOW}

The flow of process is shown in Fig. 3.

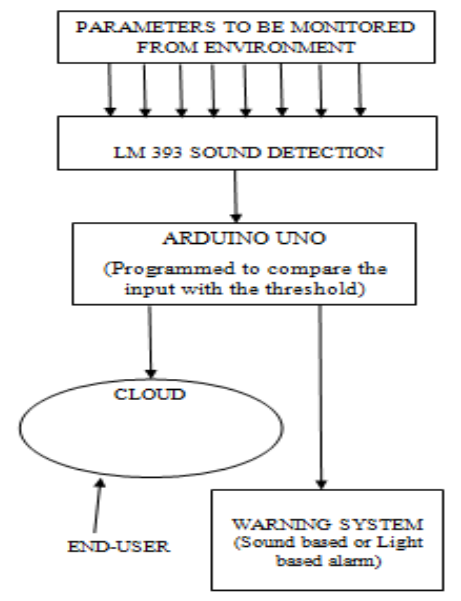

Fig. 3 Process flow diagram

This shows the process flow of the proposed system. The parameter to be measured is nothing but the sound intensity prevailing at a particular place. The LM393 sound detection sensor is the sensor used to measure sound intensity. This is given as input to Arduino uno. The Arduino Uno board is uploaded with the sketch that contains the coding to compare the input with the threshold. If the input sensed is greater than the given threshold, that is, the intensity of sound in a particular area is greater than the allowable intensity at that area, then the Arduino Microcontroller send information to the warning system. The warning system can be either Sound alarm or Light alarm. The Arduino Uno also puts the sensed data into cloud with the help of ESP8266 WiFi module. Since data is stored in cloud, User from any part of the universe can analyze the intensity of sound or the level of noise in a particular area.

\section{APPLICATIONS}

The proposed system can be used in various places where there silence needs to be maintained. Some of the places where silence monitoring is needed include libraries, schools, hospitals etc.
A. Library: Library is a public place where silence maintenance is very essential. The proposed system can be implemented at places in the library. For this application, warning system cannot be sound based. Some flash light alarm or warning systems can be used.

B. School: In schools, the proposed system can be placed in every class room. This can be helpful in monitoring the classes in the absence of the teacher. The warning system for schools can be either sound based or light based. But preferably, sound based warning system. A siren like sound may be produced when the children produce a noise higher than the threshold. Use of Sound alarm system may seem contrary but can be used within certain limits such that it does not affect the neighbours, in order to draw the attention of the children. This can also be used as an emergency alarm.

C. Hospital: Hospitals are one among the places where silence maintenance is a must. In hospitals too sound alarm warning systems cannot be used but flash light systems can be used.

\section{BLOCK DIAGRAM}

Fig. 4 shows the block diagram of the Silence monitoring system. The system consists of the sound detection sensor that sends data to the Arduino Uno. Arduino Uno sends data to the cloud through ESP8266 WiFi module. It further compares the input with the threshold and initiates the warning system when the condition given is satisfied.

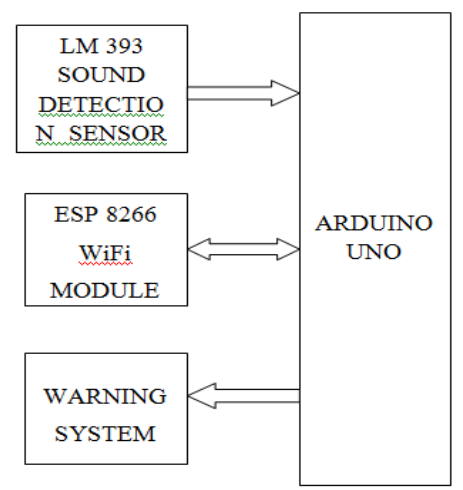

Fig. 4 Block diagram of the proposed system

\section{CIRCUIT DIAGRAM AND EXPLANATION}

ESP 8266 module and LM 393 module are connected to Arduino Uno as shown in Fig. 5.

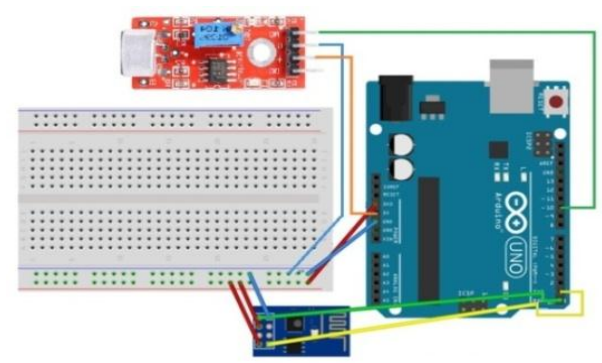

Fig. 5 Circuit diagram of the proposed system 
TABLE I CONNECTIONS

\begin{tabular}{|l|c|}
\hline \multicolumn{1}{|c|}{ Module } & Arduino Uno \\
\hline LM 393 Sound Detection Sensor & \\
\hline A0 & 10 \\
\hline Vcc or + & GV \\
\hline G & Gnd \\
\hline ESP8266 WiFi Module & Rx \\
\hline Tx & Tx \\
\hline Rx & $3.3 \mathrm{~V}$ \\
\hline CH_PD & $3.3 \mathrm{~V}$ \\
\hline VCC & Gnd \\
\hline Gnd & \\
\hline
\end{tabular}

After making all these connections the sketch is uploaded to the Arduino Uno and the outputs are obtained. To implement this in real time, the system should be battery powered and should be kept at various locations where the sound intensity is to be monitored.

\section{EXPERIMENTAL RESULTS}

The Arduino Uno board is programmed to read the analog output from the LM393 Sound detection sensor. Fig. 6 shows the analog values read by the LM 393 sensor. These data are also sent to the cloud. User from any location at any time can access this data and analyze.

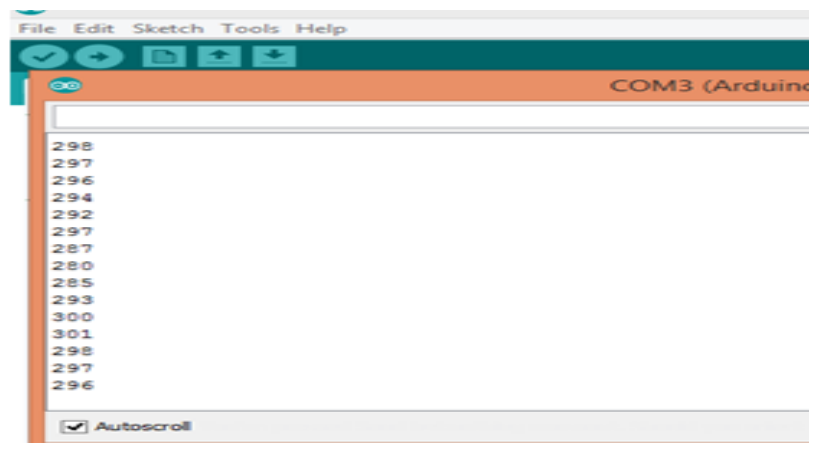

Fig. 6 Output of LM 393 in Serial monitor of Arduino

Fig. 7 shows the circuit diagram with the buzzer. The buzzer produces output only when the input from LM 393 sound detector sensors goes higher than the threshold value. Here buzzer acts as sound based warning system. Implementing in real time, the buzzer can be replaced by a siren or a flash light system.

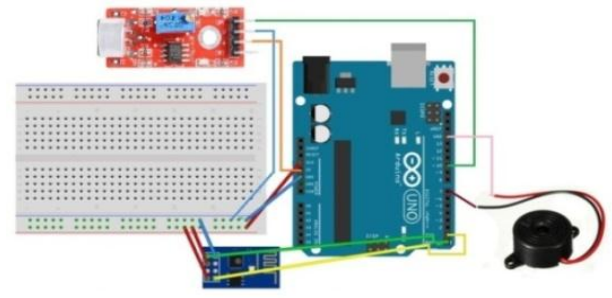

Fig. 7 Circuit diagram with the buzzer

\section{ADVANTAGES}

There are not many other such systems to monitor silence. Some of the advantages of the proposed system are as follows

1. Cost-effective

2. Easier implementation

3. Low power consumption

4. Modifications in the system can be easily made by changing the program

5. Occupies less space

6. Data can be retrieved from cloud anywhere and at any time.

\section{CONCLUSION}

With the help of this system it is possible to monitor the sound intensity at any place and since this involves storing the read data in cloud, monitoring is possible from any remote location. This system can be successfully implemented as a real time system with some necessary modifications. Implementing the silence monitoring system with Arduino Uno is found to be cost - effective. The available circuit can also be fabricated into a compact device which makes monitoring and maintenance easier. In case of Classroom silence monitoring systems in schools the data which is stored in the cloud can help to analyse the discipline of each class over time. So that the Silence record system available in schools these days can be digitized. Thereby reducing the usage of paper and reduces the efforts taken to maintain them.

\section{REFERENCES}

[1] Rajasekar, R.V. Aadhithya, B. Gopinath and M. Mohammad Jaffar Ali, "IoT based air and noise pollution monitoring system", International Journal of Advanced Research Research Trends in Engineering and Technology., Vol. 4, Special Issue 19, April 2017.

[2] Anushka Sharma, Vaishnavi Varshney, Roopank Maheshwari and Upasana Pandey, "IoT based air and sound pollution monitoring system", International Research Journal of Engineering and Technology.,Vol. 5, No. 3, March 2018.

[3] Mahantesh B. Dalawai, Siva yellampalli and S.V. Pradeep, "IoT based air and noise pollution monitoring in urban and rural areas, important zones like schools and hospitals in real time", International Digital library of Technology \& Research, Vol. 1, No. 5, May 2017.

[4] Arushi Singh, Divya Pathak, Prachi Pandit, Shruti Patil and Prof. Priti.C.Golar, "IoT based air and sound pollution monitoring system", International Journal of advanced research in Electrical, Electronics and Instrumentation Engineering, Vol. 6, No. 3, March 2017.

[5] K.G. Sindhu., H. Shruthi, M.B. Sumanth, H.M. Vijayashree, and A.P Ayesha, "IoT based air and noise pollution monitoring system", Inernational journal of Innovative research in Science, Engineering and Technology, Vol. 7, No. 5, May 2018.

[6] P. Sai Chandana, K. Sreelekha, A. Muni Likith Reddy, M. Anil Kumar Reddy, and R. Senthamilselvan, "IoT air and sound pollution monitoring system", International Journal on Applications in Engineering and Technology, Vol. 3, No. 1, pp. 18-21, March 2017.

[7] Leo Louis, "Working principle of Arduino and using it as a tool for study and research", International Journal of Control, Automation, Communication and Systems (IJCACS), Vol. 1, No. 2, April 2016.

[8] Ivan Grokhotkov, ESP8266 Arduino core documentation, Release 2.4.0, May 14, 2017.

[9] Mukesh Soni, Sound sensor module - Overview, University of Melbourne.

[10] T.H. Shivaraj Kumar, T.A. Sriraksha, and Noor U saba, "An IoT based secured smart e-campus", International Journal of Humanities and Social Science Invention, Vol. 6, No. 3, pp. 88-93, March. 2017. 